\title{
Mutações de resistência em gestantes infectadas pelo HIV: uma revisão da literatura
}

\author{
Resistance mutations in HIV-infected pregnant women: a literature review
}

\author{
Ana Teresa Mancini Pimentaa, Silvana Maria Quintanab \\ a Bióloga. Doutora em Ciências Médicas pelo Departamento de Ginecologia e Obstetrícia da Faculdade de Medicina de Ribeirão Preto da Universidade \\ de São Paulo (FMRP-USP).

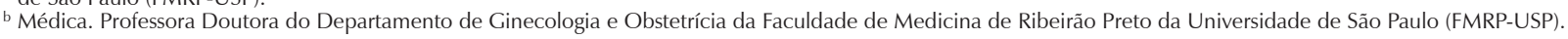 \\ Fonte de financiamento: Bolsa de estudo da CAPES.
}

RESUMO Objetivo: O objetivo desta revisão foi buscar na literatura dados sobre a prevalência de mutações de resistência do HIV aos antirretrovirais em gestantes infectadas pelo vírus.

Materiais e Métodos: A busca e seleção de artigos foi realizada nas bases de dados PubMed, Scielo e Biblioteca Virtual em Saúde sem delimitação de data. Os termos utilizados na busca foram: pregnancy, HIV e drug resistance.

Resultados: Foram encontrados 47 estudos relacionados ao assunto, realizados em 26 países, publicados no período de 1998 a 2014. A prevalência de resistência transmitida variou de zero a 18\% enquanto a resistência adquirida variou de zero a 50\% em amostras coletadas entre 1989 e 2013.

Conclusão: Generalizar os resultados é inviável devido às diferentes características dos estudos. No entanto, o monitoramento contínuo da resistência do HIV aos antirretrovirais em cada localidade é essencial.

Palavras-chave: gravidez; HIV; mutação; transmissão vertical de doença infecciosa; resistência a medicamentos.

ABSTRACT I Objetive: The aim of this review was to search the literature data on the prevalence of HIV resistance mutations to antiretroviral drugs in HIV-infected pregnant women.

Materials and methods: Articles were searched for and selected from PubMed, Scielo and Virtual Health Library electronic databases without date delimitation. Terms used in the search were as follow: pregnancy, HIV and drug resistance.

Results: There were 47 studies related to the subject conducted in 26 countries published between 1998 and 2014. The prevalence of transmitted resistance mutations varied from zero to $18 \%$, while acquired resistance varied from zero to $50 \%$ in samples collected between 1989 and 2013.

Conclusion: Generalizing results is unfeasible due to different characteristics of the studies. However, ongoing monitoring of HIV drug resistance in the localities is essential.

Keywords: pregnancy; HIV; mutation; vertical infection transmission; drug resistance. 


\section{INTRODUÇÃO}

A utilização da terapia antirretroviral profilática na prevenção da transmissão vertical do HIV é uma das medidas mais eficazes na prevenção da transmissão desse vírus, mas a presença de mutações de resistência aos antirretrovirais na população viral é capaz de diminuir a eficácia de tal medida ${ }^{1}$.

$\mathrm{O}$ uso de terapia antirretroviral associa-se com o risco de desenvolvimento de resistência aos antirretrovirais, podendo resultar em maior risco de transmissão vertical de cepas resistentes de $\mathrm{HIV}^{1,2}$, especialmente quando a gestante fez ou faz uso de terapia antirretroviral inadequadamente, facilitando a ocorrência de pressão seletiva para o desenvolvimento de vírus resistentes aos medicamentos utilizados. Existe também a possibilidade da gestante já ter adquirido cepa viral com mutações de resistência, e consequentemente transmitir cepas de HIV resistente ao feto/recém-nascido, ocorrendo futura falência terapêutica na mulher e na criança ${ }^{3,4}$. Em adição, vírus resistentes podem compor uma reserva latente em linfócitos TCD4+ em forma de pró-virus estáveis e permanecer protegidos contra a imunidade celular e contra a ação dos antirretrovirais ${ }^{5}$. Na ausência da pressão seletiva dos antirretrovirais ocorre um processo de substituição da população de vírus mutantes por vírus selvagens que apresentam maior capacidade replicativa. Quando a população de vírus mutantes é reduzida para menos de $20 \%$ a maioria dos testes genotípicos não é capaz de detectar ${ }^{6}$. Porém, com a reintrodução da terapia antirretroviral, ocorre a reaparição das cepas resistentes ${ }^{7}$. Portanto, é importante conhecer o perfil molecular das cepas de HIV das gestantes para auxiliar na melhor escolha da terapia antirretroviral e prevenir possível transmissão vertical de vírus resistentes.

O objetivo deste estudo foi revisar a literatura em busca de artigos abrangendo prevalência de mutações de resistência do HIV aos antirretrovirais em gestantes infectadas pelo vírus.

\section{MATERIAIS E MÉTODOS}

Este estudo utilizou como metodologia a busca ativa de artigos em bases de dados eletrônicas. A revisão da literatura foi realizada no mês de novembro de 2014 a partir das bases de dados PubMed (US National Library of Medicine - NIH), Scielo (Scientific Eletronic Library Online) e BVS (Biblioteca Virtual em Saúde). Com a finalidade de se delimitar o assunto, os descritores empregados na busca de artigos, de acordo com Descritores em Ciências da Saúde (DeCS) foram: pregnancy, HIV e drug resistance. Além disso, recorreu-se ao operador boleano "AND" para a combinação dos descritores. O período de publicação dos artigos não foi delimitado. $\mathrm{Na}$ base de dados Scielo a pesquisa de artigos utilizou todos os índices e foi regional. Na base de dados BVS foi utilizado todos os índices, todas as fontes e método integrado. Foram gerados 625 artigos no PubMed, 607 no BVS e 03 no Scielo, no total 1.235 estudos.

A seleção dos artigos encontrados foi realizada a partir de protocolo pré-elaborado. Estudos duplicados foram excluídos restando 677. Em seguida, 29 artigos com idioma diferente de português, inglês ou espanhol foram excluídos. Títulos foram analisados e excluídos $(n=116)$ aqueles relativos a outras doenças/infecções como malária, tuberculose, herpes e leishmaniose. Artigos de revisão, consensos e recomendações foram excluídos ( $n=175)$. A seguir, foi realizada a leitura de resumo dos estudos e/ou artigos completos. A Rede Privada Virtual (Virtual Private Network - VPN) de uma universidade pública brasileira e o portal do Pubmed foram utilizados na busca dos resumos e artigos completos. Foram excluídos artigos sem acesso livre e gratuito, comentários de outros estudos, resistência fenotípica, relato de caso, avaliação de resistência do HIV apenas no puerpério e todos aqueles que não tratavam de resistência genotípica do HIV em gestantes. Restaram 47 artigos. Na Figura 1 está descrito o processo de seleção do estudo.

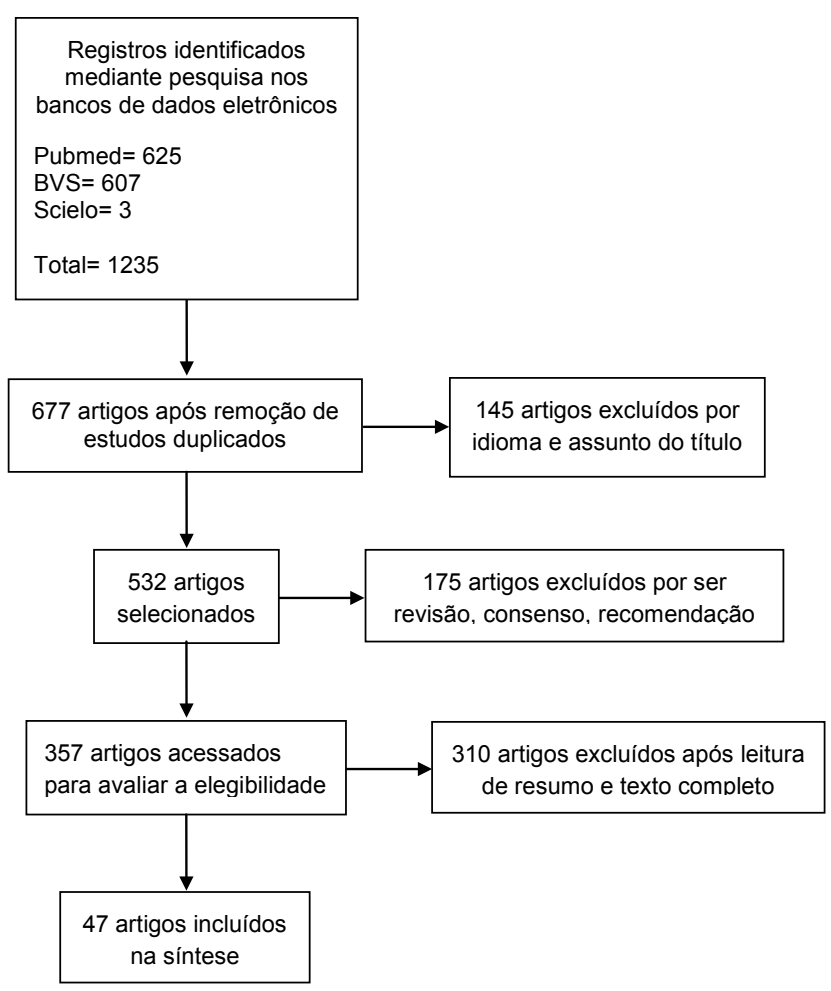

Figura 1. Fluxograma de seleção dos estudos.

\section{RESULTADOS}

Quarenta e sete artigos, representando 26 países, foram selecionados. A revisão incluiu 3717 gestantes virgens de 
tratamento e 1075 gestantes com uso prévio ou em curso de antirretrovirais. O período de coleta das amostras foi de $1989^{8}$ a $2013^{9}$. Na Tabela 1 estão apresentadas algumas características dos estudos.

O desenho de $68,0 \%$ dos trabalhos era do tipo corte transversal. A maioria dos estudos selecionados realizou a coleta das amostras durante a gestação, e em apenas quatro trabalhos a coleta foi realizada durante o parto $8,9,10,11$. A técnica mais comumente utilizada $(93,6 \%)$ para verificar a ocorrência de mutações de resistência foi o sequenciamento dos genes da protease e parte dos genes da transcriptase reversa. Outras técnicas utilizadas foram PCR utilizando alelo específico (ASPCR) com primer para detectar mutações específicas ${ }^{12,13}$ e ensaio de ligação de oligonucleotídeo (OLA) com primer para códons específicos ${ }^{14}$.
Avaliando os 47 estudos, observou-se que a ocorrência de resistência transmitida em gestantes variou de zero $^{1,13,18,21,22,24,25,30,34,35}$ a $18,0 \%{ }^{9}$, enquanto a resistência adquirida variou de zero ${ }^{10,12,22,32}$ a $50,0 \%{ }^{16}$. Este último valor de resistência adquirida foi decorrente do pequeno tamanho amostral do estudo. A prevalência total de resistência aos antirretrovirais em gestantes, considerando tanto a resistência transmitida quanto a adquirida, somou $37,5 \%$ no estudo de Rodrigues e colaboradores (2010) ${ }^{33}$, observado na Tabela 2.

As mutações mais frequentes foram: K103N (19 estudos), M184V (18 estudos) e K70R (12 estudos). No gene da protease, a mutação M46I/L foi encontrada em 14 estudos e a mutação V82A em sete Na Tabela 3 estão apresentadas as mutações de resistência observadas em cada estudo.

Tabela 1. Estudo, período de coleta das amostras, local (país) e número de amostras de gestantes virgens de tratamento ( $\mathrm{N}$-naïve) e gestantes com uso prévio ou atual de antirretrovirais (N-ARV).

\begin{tabular}{|c|c|c|c|c|c|c|c|c|c|}
\hline Estudo & Período & Local & $\mathrm{N}$-naïve & $\begin{array}{c}\text { N-uso } \\
\text { prévio de } \\
\text { ARV }\end{array}$ & Estudo & Período & Local & N-naïve & $\begin{array}{c}\text { N-uso } \\
\text { prévio de } \\
\text { ARV }\end{array}$ \\
\hline 15 & 1991-1994 & EUA e França & 61 & 15 & 30 & 1997-2005 & EUA & 22 & 22 \\
\hline 16 & 1995 & Suiça & 58 & 4 & 1 & 2003 & Brasil & 35 & 42 \\
\hline 8 & 1989-1994 & EUA & 96 & 46 & 31 & 2008-2009 & Angola & 35 & 0 \\
\hline 17 & 1991-1997 & EUA & 99 & 55 & 32 & 2006-2008 & Alemanha & 34 & 6 \\
\hline 10 & 1996-1998 & Costa do Marfim & 0 & 20 & 33 & 2007 & Brasil & 0 & 24 \\
\hline 18 & 2000 & África do Sul & 37 & 0 & 34 & 2007 & Botsuana & 71 & 0 \\
\hline 19 & $2000-2001$ & EUA & 18 & 0 & 35 & 2006-2008 & Argentina & 10 & 0 \\
\hline 20 & 2000 & Ruanda & 43 & 0 & 36 & 2009 & África do Sul & 44 & 0 \\
\hline 21 & 2000-2002 & EUA & 22 & 23 & 37 & 2007-2009 & Índia & 47 & 0 \\
\hline 14 & $?$ & EUA & 50 & 7 & 38 & 2006-2007 & Zimbabue & 236 & 0 \\
\hline 22 & 2005 & $\begin{array}{l}\text { República } \\
\text { Centro-Africana }\end{array}$ & 114 & 3 & $\begin{array}{l}39 \\
40\end{array}$ & $\begin{array}{c}2007 \\
2008-2010\end{array}$ & $\begin{array}{l}\text { Nigéria } \\
\text { Brasil }\end{array}$ & $\begin{array}{l}28 \\
17\end{array}$ & $\begin{array}{c}0 \\
66\end{array}$ \\
\hline 11 & 1997-99/2000-03 & Uganda e Malaui & 254 & 0 & 41 & 2005 e 2008 & Gabão & 107 & 0 \\
\hline 4 & $2002-2005$ & $\begin{array}{l}\text { Brasil, Argentina, } \\
\text { Bahamas e Mexico }\end{array}$ & 14 & 184 & 13 & 2008-2009 & Tanzânia & 50 & 0 \\
\hline 6 & 2004 & Brasil & 25 & 5 & 42 & 2005-2009 & África do Sul & 354 & 0 \\
\hline 12 & 1998-2004 & EUA & 89 & 0 & & 2008-2009 & Burkina Faso & 47 & 0 \\
\hline 23 & 1991-2001 & EUA & 128 & 172 & & 2009 & Malaui & 94 & 0 \\
\hline 24 & 2002 & Moçambique & 75 & 0 & 45 & 2007/2009 & Moçambique & 234 & 0 \\
\hline 25 & 2006-2007 & Uganda & 37 & 0 & $\begin{array}{c}46 \\
9\end{array}$ & $\begin{array}{l}2007-2009 \\
2008-2011\end{array}$ & $\begin{array}{l}\text { Gana } \\
\text { Argentina }\end{array}$ & $\begin{array}{l}60 \\
39\end{array}$ & $\begin{array}{c}0 \\
39\end{array}$ \\
\hline 26 & $2002-2005$ & $\begin{array}{l}\text { Brasil, Argentina, } \\
\text { Bahamas e Mexico }\end{array}$ & 43 & 154 & 47 & 2005-2008 & Brasil & 238 & 0 \\
\hline 27 & 2003-2005 & África do Sul & 240 & 120 & 48 & 2010-2012 & Tanzânia & 97 & 0 \\
\hline 28 & 2006-2007 & $\begin{array}{l}\text { Burkina Faso, Costa } \\
\text { do Marfim, Tailândia }\end{array}$ & 155 & 0 & $\begin{array}{c}9 \\
49\end{array}$ & $\begin{array}{l}2008-2013 \\
2010-2012\end{array}$ & $\begin{array}{l}\text { Argentina } \\
\text { Nigéria }\end{array}$ & $\begin{array}{l}12 \\
38\end{array}$ & $\begin{array}{l}0 \\
0\end{array}$ \\
\hline 29 & 2005-2007 & China & 80 & 0 & 50 & 2008-2010 & Brasil & 30 & 68 \\
\hline
\end{tabular}


Tabela 2. Prevalência de resistência transmitida, adquirida e total.

\begin{tabular}{|c|c|c|c|c|c|c|c|}
\hline Estudo & $\begin{array}{c}\text { Resistência } \\
\text { transmitida (\%) }\end{array}$ & $\begin{array}{c}\text { Resistência } \\
\text { adquirida (\%) }\end{array}$ & Prevalência total (\%) & Estudo & $\begin{array}{c}\text { Resistência } \\
\text { transmitida (\%) }\end{array}$ & $\begin{array}{c}\text { Resistência } \\
\text { adquirida (\%) }\end{array}$ & Prevalência total (\%) \\
\hline 15 & $1(1,6 \%)$ & $1(6,7 \%)$ & $2 / 76(2,6 \%)$ & 31 & $2(5,7 \%)$ & - & $2 / 35(5,7 \%)$ \\
\hline 16 & 4 & 2 & $6 / 62(9,6 \%)$ & 32 & $2(5,9 \%)$ & 0 & $2 / 40(5 \%)$ \\
\hline 8 & - & - & $34 / 142(24 \%)$ & 33 & - & $9(37,5 \%)$ & $9 / 24(37,5 \%)$ \\
\hline 17 & - & - & $40 / 220(18,2 \%)$ & 34 & 0 & - & 0 \\
\hline 10 & - & 0 & 0 & 35 & 0 & - & 0 \\
\hline 18 & 0 & - & 0 & 36 & $1(2,3 \%)$ & - & $1 / 44(2,3 \%)$ \\
\hline 19 & $3(17 \%)$ & - & $3 / 18(17 \%)$ & 37 & $1(2,1 \%)$ & - & $1 / 47(2,1 \%)$ \\
\hline 20 & $1(2,3 \%)$ & - & $1 / 43(2,3 \%)$ & 38 & $2(0,85 \%)$ & - & $2 / 236(0,85 \%)$ \\
\hline 21 & 0 & $11(48 \%)$ & $11 / 45(24,4 \%)$ & 39 & $3(10,7 \%)$ & - & $3 / 28(10,7 \%)$ \\
\hline 14 & - & $16(33 \%)$ & $16 / 48(33 \%)$ & 40 & $1(5,9 \%)$ & $10(15,2 \%)$ & 11/83 (13,3\%) \\
\hline 22 & 0 & 0 & 0 & 41 & $3(2,8 \%)$ & - & $3 / 107(2,8 \%)$ \\
\hline 11 & $1(0,4 \%)$ & - & $1 / 254(0,4 \%)$ & 13 & 0 & $5(10 \%)$ & $5 / 50(10 \%)$ \\
\hline 4 & - & - & $11 / 76(14,5 \%)$ & 42 & $11(3 \%)$ & - & $11 / 354(3 \%)$ \\
\hline 6 & $2(8 \%)$ & $1(20 \%)$ & $3 / 30(10 \%)$ & 43 & $3(6,4 \%)$ & - & $3 / 47(6,4 \%)$ \\
\hline 12 & $6(9,4 \%)$ & 0 & $6 / 64(9,4 \%)$ & 44 & $6(6,4 \%)$ & - & $6 / 94(6,4 \%)$ \\
\hline 23 & $11(8,6 \%)$ & $44(25,6 \%)$ & $55 / 300(18,3 \%)$ & 45 & $<5 \%-15 \%$ & - & $5 \%-15 \%$ \\
\hline 24 & 0 & - & 0 & 46 & $2(3,77 \%)$ & - & $2 / 53(3,77 \%)$ \\
\hline 25 & 0 & - & 0 & 9 & $7(18 \%)$ & $8(20 \%)$ & 15/78 (19\%) \\
\hline 26 & 3 & 4 & $7 / 55(12,7 \%)$ & 47 & $21(10,7 \%)$ & - & $21 / 197(5,6 \%)$ \\
\hline 27 & $1(0,4 \%)$ & $2(1,7 \%)$ & $3 / 360(0,8 \%)$ & 48 & $8(11,9 \%)$ & - & $8 / 67(11,9 \%)$ \\
\hline 28 & $2(1,3 \%)$ & - & $2 / 155(1,3 \%)$ & 9 & $2(16,7 \%)$ & - & $2 / 12(16,7 \%)$ \\
\hline 29 & $7(8,8 \%)$ & - & $7 / 80(8,8 \%)$ & 49 & $1(2,9 \%)$ & - & $1 / 34(2,9 \%)$ \\
\hline 30 & 0 & $10(45,5 \%)$ & $10 / 44(22,7 \%)$ & 50 & $4(13,3 \%)$ & $8(11,8 \%)$ & $12 / 98(12,2 \%)$ \\
\hline 1 & 0 & $7(16,7 \%)$ & $7 / 77(9,1 \%)$ & & & & \\
\hline
\end{tabular}

Tabela 3. Mutações de resistência nos seguimentos da transcriptase reversa e da protease.

\begin{tabular}{|c|c|c|}
\hline \multirow{2}{*}{ Estudo } & \multicolumn{2}{|c|}{ Mutações observadas } \\
\hline & Segmento da transcriptase reversa & Segmento da protease \\
\hline 15 & K70R & \\
\hline 16 & $\mathrm{~T} 215 \mathrm{Y} / \mathrm{F}$ & \\
\hline 8 & M41L, D67N, K70R, L210W, T215Y, K219Q & \\
\hline 17 & K70R, L74V, M184V, T215Y & M46I \\
\hline 19 & K103N, G190S & \\
\hline 20 & V118I & \\
\hline 21 & M41M/L, K103N, Y181C, M184V & D30N, L90M \\
\hline 14 & M41L, K70R, T215F/Y & \\
\hline 11 & V179D & \\
\hline 6 & M184V & M46I, 154V, V82A \\
\hline 12 & M184V & $\mathrm{D} 30 \mathrm{~N}$ \\
\hline 23 & $\begin{array}{l}\text { M41L, K65R, D67N, T69D, K70R,V75T, K103N,V108I,Y181C,M184V, } \\
\text { Y188L, 210W, T215Y,K219Q }\end{array}$ & $\mathrm{V} 82 \mathrm{~T} / \mathrm{A}$ \\
\hline 26 & M41L, K70R, K103N, V118I, M184V, L210W & D30N, M46I \\
\hline 27 & K103N, Y181C & \\
\hline 28 & G190A & M461 \\
\hline 29 & F77L, K103KN, V106A,V179DVE, Y181C, M184V, Y188L,T215F & \\
\hline 30 & M41L, K70R, K103N/S, M184V/I, G190A/E/Q/S, L210W, T215Y/F, & $\mathrm{M} 36 \mathrm{I} / \mathrm{L} / \mathrm{V}, \mathrm{M} 46 \mathrm{I} / \mathrm{L}, \mathrm{A} 71 \mathrm{~V} / \mathrm{I} / \mathrm{T} / \mathrm{L}, \mathrm{V} 77 \mathrm{I}, \mathrm{V} 82 \mathrm{~S} / \mathrm{A} / \mathrm{F} / \mathrm{T}, \mathrm{L} 90 \mathrm{M}$ \\
\hline 1 & M41L, D67N, K70R, K103N, E138A/K, M184V, T215Y, K219Q, P225H & D30N, M46L, V82A, N88D \\
\hline 31 & M184V, G190A & \\
\hline 32 & K103N & G48V \\
\hline 33 & D67N, K103N, V108I, E138A/G, M184V, Y188L, G190A, K219E & V32I, M46I, 147A, 154V, A71V, V82A, N88D, L90M \\
\hline
\end{tabular}


Tabela 3 (conclusão)

\begin{tabular}{|c|c|c|}
\hline \multirow{2}{*}{ Estudo } & \multicolumn{2}{|c|}{ Mutações observadas } \\
\hline & Segmento da transcriptase reversa & Segmento da protease \\
\hline 37 & K101E & \\
\hline 38 & Y181C & $185 \mathrm{~V}$ \\
\hline 39 & K101E, V179E, Y181C & \\
\hline 40 & $\begin{array}{l}\text { D } 67 N \text {, K70R, L100I, K103N, V106I,E138A, V179D,Y188L, T215Y, K219E, } \\
\text { P225H }\end{array}$ & M46L, V82A, 185V, N88D, L90M \\
\hline 41 & L210W, & L33F, M46 \\
\hline 13 & $\mathrm{~K} 70 \mathrm{R}, \mathrm{T} 215 \mathrm{~F}$ & \\
\hline 42 & K101E/P, K103N, V106M, Y181C, M184V, K219R & M461 \\
\hline 43 & K70R, K103N, Y181C, M184I, G190A, T2115I, K219E & \\
\hline 44 & D67N, K103N/S, V106A, M184V, Y188C, G190E & \\
\hline 45 & M41L, K101E, E138A/G, V179E & M46L, Q58E \\
\hline 46 & Y181C, M184V & M46L, N83D \\
\hline 9 & L100I, K101P, V106I, E138A, Y181C, Y188L, G190A & \\
\hline 47 & M41L, D67N, L74I, K101E, K103N, Y181C, M184V & $M 46 \mathrm{I} / \mathrm{L}, \mathrm{I85V}$ \\
\hline 48 & M41L, K103N/S, M184V & L89M \\
\hline 9 & K103N & \\
\hline 49 & M41L, G190A & \\
\hline 50 & K70R, K103N, Y181C, M184V, Y188L, G190A, T215Y, K219E, P225H & D30N, M46I/L, I50L, V82A, L90M \\
\hline
\end{tabular}

\section{DISCUSSÃO}

Na população de gestantes infectadas pelo HIV, incluídas nos estudos selecionados, a prevalência total de resistência aos antirretrovirais variou de zero a $37,5 \%$. Resistência transmitida foi observada em até $18 \%$ das gestantes naive e resistência adquirida em até $48 \%$ das gestantes previamente expostas a antirretrovirais. Todavia, a comparação dos resultados dos diferentes estudos é um pouco complicada devido a diferentes características das populações estudadas, os períodos de coleta das amostras e mesmo as metodologias empregadas. Além disso, a prevalência de resistência pode estar subestimada, pois o sequenciamento não é capaz de identificar variantes minoritários e a falha na amplificação de espécimes pode ser um viés, principalmente se esses espécimes apresentam mutações de resistência e baixa capacidade replicativa ${ }^{36}$.

Outra limitação deste estudo refere-se à quantidade de bases de dados eletrônicas consultadas, pois poderiam ter sido incluídas buscas em bases tais como Portal Brasileiro de Informação Científica (CAPES), Web of Science, Embase, Google Scholar, Directory of open access journals (DOAJ), World Wide Science. Com isso, pode ter havido uma subestimação de artigos potencialmente relevantes encontrados.

As principais mutações encontradas referentes às classes de antirretrovirais foram: para os inibidores nucleosídeos da transcriptase reversa (ITRN): M184I/V, K70R, T215F/Y, M41L, K219E/Q/R, D67N, L210W; para os inibidores não nucleosídeos da transcriptase reversa (ITRNN): K103N/S, Y181C, G190A/Q/S, Y188C/L, K101E/P, V106A/I/M, V179D/ $\mathrm{E} / \mathrm{V}$; e para os inibidores da protease (IP): M46I/L, V82A/F/ T/S, D30N, L90M, I85V, I54V. As mutações mais encontradas foram K103N (19 estudos) e M184V (18 estudos) que conferem resistência respectivamente a nevirapina/ efavirenz e a lamivudina, causando alto nível de resistência a essas drogas. No gene da protease as mutações mais comuns foram M46I (14 estudos) e V82A (sete estudos) com resistência, respectivamente, a indinavir e a ritonavir.

Devido às mutações intrínsecas ao próprio vírus, alguma porcentagem de HIV resistente é inevitável, mesmo com a prescrição de regimes apropriados e ótima adesão ao tratamento antirretrovira|43,44. Com isso, há preocupações quanto a limitar futuras opções terapêuticas para as mulheres com uso prévio de terapia antirretroviral profilática para transmissão vertical devido à emergência de mutações de resistência ${ }^{4,32}$. A emergência de cepas resistentes decorrente da pressão seletiva do uso de terapias antirretrovirais tem sido potencializada pelo aumento do acesso a regimes de tratamento ${ }^{42}$, gerando inquietação quanto ao aumento da prevalência de resistência transmitida devido à transmissão de vírus resistentes. A dose única de nevirapina durante o trabalho de parto reduz a transmissão vertical do HIV, mas induz o aparecimento de cepas de vírus resistentes a essa droga $^{5}$. E mesmo o uso de esquema triplo por tempo limitado é capaz de induzir o aparecimento de cepas resistentes aos antirretrovirais ${ }^{51}$. Assim sendo, é importante o monitoramento da prevalência de mutações de resistência a antirretrovirais e 
da variabilidade genética do HIV com o objetivo de prevenir a transmissão de cepas resistentes, de conhecer a necessidade de desenvolvimento e introdução de novas drogas antirretrovirais e para o melhor entendimento do impacto da variabilidade genética do HIV no diagnóstico e prognóstico desta infecção nas diferentes regiões de cada país ${ }^{52}$.

Transmissões perinatais de vírus com mutações de resistência já foram descritas ${ }^{2,13,53}$. Contudo, há poucas evidências de que a presença de vírus mutantes aumente o risco de transmissão vertical quando as recomendações profiláticas são adotadas adequadamente ${ }^{15,16}$. Em cada evento de transmissão do HIV ocorre um rigoroso gargalo genético, desse modo, a população viral da pessoa infectada deriva de uma única variante do transmissor ${ }^{54}$. Por outro lado, é possível acontecer a transmissão de múltiplas variantes ${ }^{55}$, sugerindo que o gargalo genético não é inteiramente limitante. Na transmissão vertical, vírus transmitidos intraútro tendem a ser de uma única variante materna predominante no plasma, enquanto vírus transmitidos intraparto tendem a ser de múltiplas variantes ${ }^{56}$. Contudo, ressalta-se que os esforços para prevenir a emergência de resistência devido à profilaxia para transmissão vertical devem ser maximizados ${ }^{32}$.

Em uma coorte francesa, a transmissão vertical do HIV em nascimentos a termo era dez vezes maior quando a carga viral materna próxima ao parto estava acima de 10.000 cópias/ $\mathrm{mL}^{57}$. O risco da transmissão vertical é maior em gestantes com carga viral elevada e baixa contagem de linfócitos $\mathrm{T} \mathrm{CD} 4+{ }^{58}$. Deste modo, sugere-se que o uso adequado de antirretrovirais seja capaz de reduzir a carga viral das gestantes e aumentar a contagem de linfócitos T CD4+, e consequentemente diminuir o risco da transmissão perinatal do vírus, com mutações de resistência ou não. Somado a isso, boa qualidade do serviço de saúde na atenção às gestantes certamente é fator importante na ausência de transmissão vertical do HIV. Portanto, mais importante é o início precoce do pré-natal, boa adesão ao pré-natal e uso apropriado dos medicamentos prescritos.

No Brasil, o teste genotípico passou a ser indicado para gestantes infectadas pelo HIV a partir de 2013, porém é preciso avaliar os benefícios de sua utilização, assim como o potencial retardo do início da terapia antirretroviral e também avaliar a prevalência de resistência no país ${ }^{59}$. A genotipagem para pacientes naive é uma estratégia que pode conduzir a escolha de um regime antirretroviral inicial mais eficaz e provavelmente de sobrevida mais longa para pacientes que apresentam vírus com mutações de resistência aos antirretrovirais. O teste proporciona benefício para aquelas que apresentam resistência transmitida e não tanto para aquelas que não apresentam resistência ${ }^{60}$. Já o teste aplicado para pacientes com uso prévio de antirretrovirais, auxilia na escolha da terapia, resultando em melhor resultado virológico para a paciente, comparado com a escolha de regimes baseados na história clínica e terapêutica ${ }^{61}$.

Em conclusão, uma generalização dos resultados é inviável devido às diferentes características dos estudos. Assim, o monitoramento contínuo dos níveis de resistência do HIV aos antirretrovirais em cada localidade é essencial para determinar ações prioritárias no cuidado da saúde das gestantes infectadas pelo HIV e otimizar os esquemas de tratamento vigentes, reduzindo a emergência de cepas resistentes.

\section{REFERÊNCIAS}

1. Machado ES, Afonso AO, Nissley DV, Lemey P, Cunha SM, Oliveira $\mathrm{RH}$, Soares MA. Emergency of primary NNRTI resistance mutations without antiretroviral selective pressure in a HAART-treated child. PLoS One. 2009;4(3):e4806. http://dx.doi.org/10.1371/journal. pone.0004806

2. Cardoso LP, Pereira GA, Viegas AA, Schmaltz LE, Stefani MM. HIV-1 primary and secondary antiretroviral drug resistance and genetic diversity among pregnant women from central Brazil. J Med Virol. 2010 Mar;82(3):351-7. http://dx.doi.org/10.1002/jmv.21722

3. Larbalestier N, Mullen J, O'Shea S, Cuttam F, Sabin CA, Chrystie IL, Welch J, Zuckerman M, Hay P, Rice P, Taylor GP, de Ruiter A. Drug resistance is uncommon in pregnant women with low viral loads taking zidovudine monotherapy to prevent perinatal HIV transmission. AIDS. 2003;17:2665-7. http://dx.doi. org/10.1097/00002030-200312050-00015

4. Duran AS, Losso MH, Salomón H, Harris DR, Pampuro S, SotoRamirez LE, Duarte G, de Souza RS, Read JS; NISDI Perinatal Study Group. Drug resistance among HIV-infected pregnant women receiving antiretrovirals for prophylaxis. AIDS. 2007;21(2):199-205. http://dx.doi.org/10.1097/QAD.0b013e328011770b

5. Wind-Rotolo M, Durand C, Cranmer L, Reid A, Martinson N, Doherty M, Jilek BL, Kagaayi J, Kizza A, Pillay V, Laeyendecker O, Reynolds SJ, Eshleman SH, Lau B, Ray SC, Siliciano JD, Quinn TC, Siliciano RF. Identification of nevirapine-resistant HIV-1 in the latent reservoir after single-dose nevirapine to prevent mother-to-child transmission of HIV-1. J Infect Dis. 2009;199(9):1301-9. http:// dx.doi.org/10.1086/597759

6. Kakehasi FM, Tupinambás U, Cleto S, Aleixo A, Lin E, Melo VH, Aguiar RA, Pinto JA. Persistence of genotypic resistance to nelfinavir among women exposed to prophylactic antiretroviral therapy during pregnancy. AIDS Res Hum Retroviruses. 2007;23(12):1515-20. http://dx.doi.org/10.1089/aid.2007.0025

7. Afani A, Ayala M, Meyer A, Cabrera R, Acevedo W. Resistencia primaria a terapia antirretroviral en pacientes con infección por $\mathrm{VIH} /$ SIDA en Chile. Rev Med Chil. 2005;133(3):295-301. http://dx.doi. org/10.4067/S0034-98872005000300004

8. Welles SL, Pitt J, Colgrove R, McIntosh K, Chung PH, Colson A, Lockman S, Fowler MG, Hanson C, Landesman S, Moye J, Rich KC, Zorrilla C, Japour AJ. HIV-1 genotypic zidovudine drug resistance and the risk of maternal-infant transmission in the women and infants transmission study. The Women and Infants Transmission Study Group. AIDS. 2000;14(3):263-71. http://dx.doi. org/10.1097/00002030-200002180-00008 
9. Cecchini D, Zapiola I, Giuliano SF, Martinez M, Rodriguez C, Bouzas MB. Transmitted drug resistance in women with intrapartum HIV-1 diagnosis: a pilot epidemiological survey in Buenos Aires, Argentina. J Int AIDS Soc. 2014;17(4 Suppl 3):19704. http://dx.doi. org/10.7448/ias.17.4.19704

10. Ekpini RA, Nkengasong JN, Sibailly T, Maurice C, Adjé C, Monga BB, Roels TH, Greenberg AE, Wiktor SZ. Changes in plasma HIV-1-RNA viral load and CD4 cell counts, and lack of zidovudine resistance among pregnant women receiving short-course zidovudine. AIDS. 2002;16(4):625-30. http://dx.doi.org/10.1097/00002030200203080-00015

11. Church JD, Hudelson SE, Guay LA, Chen S, Hoover DR, Parkin $\mathrm{N}$, Fiscus SA, Mmiro F, Musoke P, Kumwenda N, Jackson JB, Taha $\mathrm{TE}$, Eshleman SH. HIV type 1 variants with nevirapine resistance mutations are rarely detected in antiretroviral drug-naive African women with subtypes A, C, and D. AIDS Res Hum Retroviruses. 2007;23(6):764-8. http://dx.doi.org/10.1089/aid.2006.0272

12. Paredes R, Cheng I, Kuritzkes DR, Tuomala RE. High prevalence of primary lamivudine and nelfinavir resistance in HIV-1infected pregnant women in the United States, 1998-2004. AIDS. 2007;21(15):2103-6. http://dx.doi.org/10.1097/QAD. ob013e3282ef3822

13. Hauser A, Sewangi J, Mbezi P, Dugange F, Lau I, Ziske J, Theuring S, Kuecherer C, Harms G, Kunz A. Emergence of minor drug-resistant HIV-1 variants after triple antiretroviral prophylaxis for prevention of vertical HIV-1 transmission. PLos One. 2012;7:e32055. http:// dx.doi.org/10.1371/journal.pone.0032055

14. Frenkel LM, McKernan J, Dinh PV, Goldman D, Hitti J, Watts DH, Cooper ER, Dragavon J, Coombs RW. HIV type 1 zidovudine (ZDV) resistance in blood and uterine cervical secretions of pregnant women. AIDS Res Hum Retroviruses. 2006;22(9):870-3. http:// dx.doi.org/10.1089/aid.2006.22.870

15. Eastman PS, Shapiro DE, Coombs RW, Frenkel LM, McSherry GD, Britto P, Herman SA, Sperling RS. Maternal viral genotypic zidovudine resistance and infrequent failure of zidovudine therapy to prevent perinatal transmission of human immunodeficiency virus type 1 in pediatric AIDS Clinical Trials Group Protocol 076. J Infect Dis. 1998;177(3):557-64. http://dx.doi.org/10.1086/514228

16. Kully C, Yerly S, Erb P, Kind C, Krautheim A, Perrin L, Rudin C. Codon 215 mutations in human immunodeficiency virus-infected pregnant women. Swiss Collaborative 'HIV and Pregnancy' Study. J Infect Dis. 1999;179(3):705-8. http://dx.doi.org/10.1086/314615

17. Palumbo P, Holland B, Dobbs T, Pau CP, Luo CC, Abrams EJ, Nesheim S, Vink P, Respess R, Bulterys M. Perinatal AIDS Collaborative Transmission Study. Antiretroviral resistance mutations among pregnant human immunodeficiency virus type 1-infected women and their newborns in the United States: vertical transmission and clades. J Infect Dis. 2001;184(9):1120-6. http://dx.doi. org/10.1086/323804

18. Pillay C, Bredell H, McIntyre J, Gray G, Morris L. HIV-1 subtype C reverse transcriptase sequences from drug-naive pregnant women in South Africa. AIDS Res Hum Retroviruses. 2002;18(8):605-10. http://dx.doi.org/10.1089/088922202753747950

19. Juethner SN, Williamson C, Ristig MB, Tebas P, Seyfried W, Aberg JA.egnant women. J Acquir Immune Defic Syndr. 2003;32(2):153-6. http://dx.doi.org/10.1097/00126334-200302010-00006
20. Servais J, Lambert C, Karita E, Vanhove D, Fischer A, Baurith T, Schmit JC, Schneider F, Hemmer R, Arendt V. HIV type 1 pol gene diversity and archived nevirapine resistance mutation in pregnant women in Rwanda. AIDS Res Hum Retroviruses. 2004 Mar;20(3):279-83. http://dx.doi.org/10.1089/088922204322996518

21. Shah SS, Crane M, Monaghan K, McGowan JP. Genotypic resistance testing in HIV-infected pregnant women in an urban setting. Int J STD AIDS. 2004 Jun;15(6):384-7. http://dx.doi. org/10.1258/095646204774195236

22. Marechal V, Jauvin V, Selekon B, Leal J, Pelembi P, Fikouma V, Gabrie P, Heredeibona LS, Goumba C, Serdouma E, Ayouba A, Fleury H. Increasing HIV type 1 polymorphic diversity but no resistance to antiretroviral drugs in untreated patients from Central African Republic: a 2005 study. AIDS Res Hum Retroviruses. 2006;22(10):1036-44. http://dx.doi.org/10.1089/ aid.2006.22.1036

23. Welles SL, Bauer GR, LaRussa PS, Colgrove RC, Pitt J. Time trends for HIV-1 antiretroviral resistance among antiretroviral-experienced and naive pregnant women in New York City during 1991 to early 2001. J Acquir Immune Defic Syndr. 2007;44(3):329-35. http:// dx.doi.org/10.1097/QAl.0b013e31802f1296

24. Abreu CM, Brindeiro PA, Martins AN, Arruda MB, Bule E, Stakteas S, Tanuri A, de Moraes Brindeiro R. Genotypic and phenotypic characterization of human immunodeficiency virus type 1 isolates circulating in pregnant women from Mozambique. Arch Virol. 2008; 153(11):2013-7. http://dx.doi.org/10.1007/s00705-008-0215-6

25. Ndembi N, Lyagoba F, Nanteza B, Kushemererwa G, Serwanga J, Katongole-Mbidde E, Grosskurth H, Kaleebu P. Uganda HIV Drug Resistance Working Group. Transmitted antiretroviral drug resistance surveillance among newly HIV type 1-diagnosed women attending an antenatal clinic in Entebbe, Uganda. AIDS Res Hum Retroviruses. 2008; 24(6): 889-95. http://dx.doi.org/10.1089/aid.2007.0317

26. Soto-Ramirez LE, Rodriguez-Diaz R, Durán AS, Losso $M H$, Salomón H, Gómez-Carrillo M, Pampuro S, Harris DR, Duarte G, De Souza RS, Read JS; NISDI Perinatal Study Group. Antiretroviral resistance among HIV type 1 -infected women first exposed to antiretrovirals during pregnancy: plasma versus PBMCs. AIDS Res Hum Retroviruses. 2008;24(6):797-804. http://dx.doi.org/10.1089/ aid. 2007.0246

27. Martinson NA, Morris L, Johnson J, Gray GE, Pillay V, Ledwaba J, Dhlamini P, Cohen S, Puren A, Steyn J, Heneine W, McIntyre JA. Women exposed to single-dose nevirapine in successive pregnancies: effectiveness and nonnucleoside reverse transcriptase inhibitor resistance. AIDS. 2009;23(7):809-16. http://dx.doi. org/10.1097/QAD.0b013e328323ad49

28. Ayouba A, Lien TT, Nouhin J, Vergne L, Aghokeng AF, Ngo-GiangHuong N, Diop H, Kane CT, Valéa D, Rouet F, Joulia-Ekaza D, Toni TD, Nerrienet E, Ngole EM, Delaporte E, Costagliola D, Peeters M, Chaix ML. Low prevalence of HIV type 1 drug resistance mutations in untreated, recently infected patients from Burkina Faso, Côte d'Ivoire, Senegal, Thailand, and Vietnam: the ANRS 12134 study. AIDS Res Hum Retroviruses. 2009;25(11):1193-6. http://dx.doi. org/10.1089/aid.2009.0142

29. Han J, Wang L, Jiang Y, Zhang Q, Fang L, Yao J, Wang Q. Resistance mutations in HIV-1 infected pregnant women and their infants receiving antiretrovirals to prevent HIV-1 vertical transmission in China. Int J STD AIDS. 2009;20(4):249-54. http://dx.doi. org/10.1258/ijsa.2008.008480 
30. Weinberg A, Forster-Harwood J, McFarland EJ, Pappas J, Davies JK, Kinzie K, Barr EA, Paul SM, Salbenblatt CR, Soda E, Vazquez A, Levin MJ. Resistance to antiretrovirals in HIV-infected pregnant women. J Clin Virol. 2009;45(1):39-42. http://dx.doi.org/10.1016/j. jcv.2009.02.009

31. Castelbranco EP, da Silva Souza E, Cavalcanti AM, Martins AN, de Alencar LC, Tanuri A. Frequency of primary resistance to antiretroviral drugs and genetic variability of HIV-1 among infected pregnant women recently diagnosed in Luanda-Angola. AIDS Res Hum Retroviruses. 2010;26(12):1313-6. http://dx.doi.org/10.1089/ aid.2010.0111

32. Gingelmaier A, Eberle J, Kost BP, Bogner JR, Hofmann J, Weissenbacher T, Kästner R, Friese K, Weizsaecker K. Protease inhibitor-based antiretroviral prophylaxis during pregnancy and the development of drug resistance. Clin Infect Dis. 2010 Mar 15;50(6):890-4. http://dx.doi.org/10.1086/650747

33. Rodrigues R, Manenti S, Romao PRT, Ferreira JLP, Batista JPG, Siqueira AFAC, de Macedo Brigido LF. Young pregnant women living with HIV/ AIDS in Criciuma, Southern Brazil, are infected almost exclusively with HIV type 1 clade C. AIDS Res Hum Retroviruses. 2010;26:351-7. http://dx.doi.org/10.1089/aid.2009.0214

34. Bussmann, $\mathrm{H}$, de la Hoz Gomez F, Roels TH, Wester CW, Bodika SM, Moyo S, Taffa N, Anderson MG, Mine M, Bile EC, Yang C, Mphoyakgosi K, Lehotzky EA, Mlotshwa B, Mmelesi M, Seipone K, Makhema MJ, Marlink RG, Novitsky V, Essex M. Prevalence of transmitted HIV drug resistance in Botswana: lessons learned from the HIVDR-Threshold Survey conducted among women presenting for routine antenatal care as part of the 2007 National Sentinel Survey. AIDS Res Hum Retroviruses. 2011;27(4):365-72. http:// dx.doi.org/10.1089/aid.2009.0299

35. Pando MA, Gómez-Carrillo M, Vignoles M, Rubio AE, dos Ramos Farias MS, Vila M, Rossi D, Ralón G, Marone R, Reynaga E, Sosa J, Torres O, Maestri M, Avila MM, Salomón H. Incidence of HIV type 1 infection, antiretroviral drug resistance, and molecular characterization in newly diagnosed individuals in Argentina: a Global Fund Project. AIDS Res Hum Retroviruses. 2011;27(1): 17-23. http://dx.doi.org/10.1089/aid.2010.0013

36. Parboosing R, Naidoo A, Gordon M, Taylor M, Vella V. Resistance to antiretroviral drugs in newly diagnosed, young treatment-naïve HIVpositive pregnant women in the Province of KwaZulu-Natal, South Africa. J Med Virol. 2011;83(9):1508-13. http://dx.doi.org/10.1002/ jmv. 22143

37. Thorat SR, Chaturbhuj DN, Hingankar NK, Chandrasekhar V, Koppada R, Datkar SR, Srikantiah P, Garg R, Kabra S, Haldar P, Reddy DC, Bachani D, Tripathy SP, Paranjape RS. Surveillance of transmitted HIV type 1 drug resistance among HIV type 1-positive women attending an antenatal clinic in Kakinada, India. AIDS Res Hum Retroviruses. 2011 Dec;27(12):1291-7. http://dx.doi. org/10.1089/aid.2011.0036

38. Tshabalala M, Manasa J, Zijenah LS, Rusakaniko S, Kadzirange G, Mucheche M, Kassaye S, Johnston E, Katzenstein D. Surveillance of transmitted antiretroviral drug resistance among HIV-1 infected women attending antenatal clinics in Chitungwiza, Zimbabwe. PLoS One. 2011;6(6):e21241. http://dx.doi.org/10.1371/journal. pone.0021241

39. Ajoge HO, Gordon ML, Ibrahim S, Shittu OS, Ndung'u T, Olonitola SO. Drug resistance pattern of HIV type 1 isolates sampled in 2007 from therapy-naive pregnant women in North-Central Nigeria. AIDS Res Hum Retroviruses. 2012 Jan;28(1):115-8. http://dx.doi. org/10.1089/aid.2011.0115

40. Alcântara KC, Lins JB, Albuquerque M, Aires LM, Cardoso LP, Minuzzi AL, Stefani MM. HIV-1 mother-to-child transmission and drug resistance among Brazilian pregnant women with high access to diagnosis and prophylactic measures. J Clin Virol. 2012;54(1):1520. http://dx.doi.org/10.1016/j.jcv.2012.01.011

41. Caron M, Lekana-Douki SE, Makuwa M, Obiang-Ndong GP, Biba O, Nkoghé D, Kazanji M. Prevalence, genetic diversity and antiretroviral drugs resistance-associated mutations among untreated HIV-1infected pregnant women in Gabon, central Africa. BMC Infect Dis. 2012;12:64. http://dx.doi.org/10.1186/1471-2334-12-64

42. Hunt GM, Ledwaba J, Basson AE, Moyes J, Cohen C, Singh B, Bertagnolio S, Jordan MR, Puren A, Morris L. Surveillance of transmitted HIV-1 drug resistance in Gauteng and KwaZulu-Natal Provinces, South Africa, 2005-2009. Clin Infect Dis. 2012;54 Suppl 4:S334-8. http://dx.doi.org/10.1093/cid/cir1017

43. Somda A, Sangare L, Soro M, Yameogo S, Bazie B, Bigirimana F, Bertagnolio S, Peeters M, Mouacha F, Rivera AM, Jordan MR, Sanou MJ. Surveillance of transmitted drug-resistant HIV among young pregnant women in Ouagadougou, Burkina Faso. Clin Infect Dis. 2012;54 Suppl 4:S317-9. http://dx.doi.org/10.1093/cid/cir988

44. Wadonda-Kabondo N, Banda R, Moyo K, M'bang'ombe M, Chiwaula M, Porter C, Jordan MR. Prevalence of transmitted HIV drug resistance among newly diagnosed antiretroviral therapynaive pregnant women in Lilongwe and Blantyre, Malawi. Clin Infect Dis. 2012;54 Suppl 4:S324-7. http://dx.doi.org/10.1093/cid/ cir993

45. Bila DC, Young P, Merks H, Vubil AS, Mahomed M, Augusto A, Abreu CM, Mabunda NJ, Brooks JI, Tanuri A, Jani IV. Evolution of primary HIV drug resistance in a subtype $\mathrm{C}$ dominated epidemic in Mozambique. PLoS One. 2013;8(7):e68213. http://dx.doi. org/10.1371/journal.pone.0068213

46. Bonney EY, Addo NA, Ntim NA, Addo-Yobo F, Bondzie P, Aryee KE, Barnor J, Brandful J, Bekoe V, Ohene SA, Ampofo W. Low level of transmitted HIV Drug resistance at two HIV care centres in Ghana: a threshold survey. Ghana Med J. 2013;47(2):82-6.

47. Pilotto JH, Grinsztejn B, Veloso VG, Velasque LS, Friedman RK, Moreira RI, Rodrigues-Pedro A, Oliveira SM, Currier JS, Morgado MG. Moderate prevalence of transmitted drug resistance mutations among antiretroviral-naive HIV-infected pregnant women in Rio de Janeiro, Brazil. AIDS Res Hum Retroviruses. 2013;29(4):681-6. http://dx.doi.org/10.1089/aid.2011.0333

48. Vairo F, Nicastri E, Liuzzi G, Chaula Z, Nguhuni B, Bevilacqua N, Forbici F, Amendola A, Fabeni L, De Nardo P, Perno CF, Cannas A, Sakhoo C, Capobianchi MR, Ippolito G; AMANI Study Group. HIV-1 drug resistance in recently HIV-infected pregnant mother's naïve to antiretroviral therapy in Dodoma urban, Tanzania. BMC Infect Dis. 2013;13:439. http://dx.doi.org/10.1186/1471-2334-13-439

49. Imade GE1, Sagay AS, Chaplin B, Chebu P, Musa J, Okpokwu J, Hamel DJ, Pam IC, Agbaji O, Samuels J, Meloni S, Sankale JL, Okonkwo P, Kanki P. Short communication: Transmitted HIV drug resistance in antiretroviral-naive pregnant women in north central Nigeria. AIDS Res Hum Retroviruses. 2014;30(2):127-33. http:// dx.doi.org/10.1089/aid.2013.0074 
50. Reis MN, de Alcântara KC, Cardoso LP, Stefani MM. Polymorphisms in the HIV-1 gp41 env gene, natural resistance to enfuvirtide (T-20) and pol resistance among pregnant Brazilian women. J Med Virol. 2014 Jan;86(1):8-17. http://dx.doi.org/10.1002/jmv.23738

51. Pilotto JH, Grinsztejn B, Veloso V, Couto-Fernandez JC, RodriguesPedro A, Velaso-de-Castro CA, Eurico Ribeiro J, Khalili R, Muri S, Ismerio R, Hoffman R, Currier J, Morgado MG. HIV drug resistance after HAART discontinuation among treatment naive women who initiated antiretroviral therapy for the prevention of mother-to-child transmission in Rio de Janeiro, Brazil. Antivir Ther. 2009;14(suppl 1):A167 (abstract no 144).

52. Westin MR, Biscione FM, Fonseca M, Ordones M, Rodrigues $M$, Greco DB, Tupinambas U. Resistance-associates mutation prevalence according to subtypes B and non-B of HIV type 1 in antiretroviral-experienced patients in Minas Gerais, Brazil. AIDS Res Hum Retroviruses. 2011;27(9):981-7. http://dx.doi.org/10.1089/ aid.2010.0260

53. Delaugerre C, Chaix ML, Blanche S, Warszawski J, Cornet D, Dollfus C, Schneider V, Burgard M, Faye A, Mandelbrot L, Tubiana R, Rouzioux C; ANRS French Perinatal Cohort. Perinatal acquisition of drugresistant HIV-1 infection: mechanism and long-term outcome. Retrovirology. 2009;6:85. http://dx.doi.org/10.1186/1742-4690-6-85

54. Haaland RE, Hawkins PA, Salazar-Gonzalez J, Johnson A, Tichacek A, Karita E, Manigart O, Mulenga J, Keele BF, Shaw GM, Hahn BH, Allen SA, Derdeyn CA, Hunter E. Inflammatory Genital Infections Mitigate a Severe Genetic Bottleneck in Heterosexual Transmission of Subtype A and C HIV-1. PLoS Pathog. 2009;5(1):e1000274. http://dx.doi.org/10.1371/journal.ppat.1000274

55. Pasquier C, Cayrou C, Blancher A, Tourne-Petheil C, Berrebi A, Tricoire J, Puel J, Izopet J. Molecular evidence for mother-to-child transmission of multiple variants by analysis of RNA and DNA sequences of human immunodeficiency virus type 1. J Virol. 1998;72(11):8493-501.

56. Kwiek JJ, Russell ES, Dang KK, Burch CL, Mwapasa V, Meshnick SR, Swanstrom R. The molecular epidemiology of HIV-1 envelope diversity during HIV-1 subtype $\mathrm{C}$ vertical transmission in Malawian mother-infant pairs. AIDS. 2008;22(7):863-71. http://dx.doi. org/10.1097/QAD.0b013e3282f51ea0

57. Warszawski J, Tubiana R, Le Chenadec J, Blanche S, Teglas JP, Dollfus C, Faye A, Burgard M, Rouzioux C, Mandelbrot L; ANRS French Perinatal Cohort. Mother-to-child HIV transmission despite antiretroviral therapy in the ANRS French Perinatal Cohort. AIDS. 2008;22(2):289-99. http://dx.doi.org/10.1097/ QAD.0b013e3282f3d63c

58. Kourtis AP, Bulterys M. Mother-to-child transmission of HIV: pathogenesis, mechanism and pathways. Clin Perinatol. 2010;37(4):721-37. http://dx.doi.org/10.1016/j.clp.2010.08.004

59. Ministério da Saúde (BR). Secretaria de Vigilância em Saúde, Programa Nacional de DST e Aids. Protocolo clínico e diretrizes terapêuticas para adultos vivendo com HIV/ aids. Versão preliminar. Brasília: Ministério da Saúde; 2013. 75 p.

60. Sax PE, Islam R, Walensky RP, Losina E, Weinstein MC, Goldie SJ, Sadownik SN, Freedberg KA. Should Resistance testing be performed for treatment-naive HIV-infected patients? A Costeffectiveness analysis. Clin Infect Dis. 2005;41(9):1316-23. http:// dx.doi.org/10.1086/496984

61. Tural C, Ruiz L, Holtzer C, Schapiro J, Viciana P, González J, Domingo P, Boucher C, Rey-Joly C, Clotet B; Havana Study Group. Clinical utility of HIV-1 genotyping and expert advice: the Havana Trial. AIDS. 2002;16(2):209-18. http://dx.doi.org/10.1097/00002030200201250-00010 